\title{
Anomalous Hall effect in a two-dimensional electron gas
}

\author{
Tamara S. Nunner, ${ }^{1}$ N. A. Sinitsyn, ${ }^{2,3}$ Mario F. Borunda, ${ }^{2}$ V. K. Dugaev, ${ }^{4}$ A. A. Kovalev, ${ }^{2}$ Ar. Abanov, ${ }^{2}$ Carsten Timm, ${ }^{5}$ \\ T. Jungwirth, ${ }^{6,7}$ Jun-ichiro Inoue, ${ }^{8}$ A. H. MacDonald, ${ }^{9}$ and Jairo Sinova ${ }^{2,6}$ \\ ${ }^{1}$ Institut für Theoretische Physik, Freie Universität Berlin, Arnimallee 14, 14195 Berlin, Germany \\ ${ }^{2}$ Department of Physics, Texas A\&M University, College Station, Texas 77843-4242, USA \\ ${ }^{3}$ CNLS/CCS-3, Los Alamos National Laboratory, Los Alamos, New Mexico 87544, USA \\ ${ }^{4}$ Department of Mathematics and Applied Physics, Rzeszów University of Technology, Aleja Powstanców Warszawy 6, \\ 35-959 Rzeszów, Poland \\ ${ }^{5}$ Department of Physics and Astronomy, University of Kansas, Lawrence, Kansas 66045, USA \\ ${ }^{6}$ Institute of Physics ASCR, Cukrovarnická 10, 16253 Praha 6, Czech Republic \\ ${ }^{7}$ School of Physics and Astronomy, University of Nottingham, Nottingham NG7 2RD, United Kingdom \\ ${ }^{8}$ Department of Applied Physics, Nagoya University, Nagoya 464-8603, Japan \\ ${ }^{9}$ Department of Physics, University of Texas at Austin, Austin, Texas 78712-1081, USA
}

(Received 1 June 2007; published 13 December 2007)

\begin{abstract}
The anomalous Hall effect in a magnetic two-dimensional electron gas with Rashba spin-orbit coupling is studied within the Kubo-Streda formalism in the presence of pointlike potential impurities. We find that all contributions to the anomalous Hall conductivity vanish to leading order in disorder strength when both chiral subbands are occupied. In the situation that only the majority subband is occupied, all terms are finite in the weak scattering limit and the total anomalous Hall conductivity is dominated by skew scattering. We compare our results to previous treatments and resolve some of the discrepancies present in the literature.
\end{abstract}

DOI: 10.1103/PhysRevB.76.235312

PACS number(s): 72.15.Eb, 72.20.Dp

\section{INTRODUCTION}

In 1879, Hall ran a current through a gold foil and discovered that a transverse voltage was induced when the film was exposed to a perpendicular magnetic field. ${ }^{1}$ The ratio of this Hall voltage to the current density is the Hall resistivity. For paramagnetic materials, the Hall resistivity is proportional to the applied magnetic field, and Hall measurements give information about the concentration of free carriers and determine whether they are holes or electrons. Magnetic films exhibit both this ordinary Hall response and an extraordinary or anomalous Hall response that does not disappear at zero magnetic field and is proportional to the internal magnetization: $R_{\text {Hall }}=R_{O} H+R_{S} M$, where $R_{\text {Hall }}$ is the Hall resistance, $R_{o}$ and $R_{s}$ are the ordinary and anomalous Hall coefficients, $M$ is the magnetization, and $H$ is the applied magnetic field. The anomalous Hall effect (AHE) is the consequence of spin-orbit coupling and allows an indirect measurement of the internal magnetization.

Despite the simplicity of the experiment, the theoretical basis of the AHE is still hotly debated and a source of conflicting reports. ${ }^{2}$ Different mechanisms contribute to the AHE: an intrinsic mechanism and extrinsic mechanisms such as skew-scattering and side-jump contributions. The intrinsic mechanism is based solely on the topological properties of the Bloch states originating from the spin-orbit-coupled electronic structure as first suggested by Karplus and Luttinger. ${ }^{3}$ Their approach gives an anomalous Hall coefficient $R_{s}$ proportional to the square of the ordinary resistivity, since the intrinsic AHE itself is insensitive to impurities. The skewscattering mechanism, as first proposed by Smit, ${ }^{4,5}$ relies on an asymmetric scattering of the conduction electrons by impurities present in the material. Not surprisingly, this skewscattering contribution to $R_{s}$ is sensitive to the type and range of the scattering potential and, in contrast to the intrinsic mechanism, scales linearly with the diagonal resistivity. The presence of impurities also leads to a side-step type of scattering, which contributes to a net current perpendicular to the initial momentum. This is the so-called side-jump contribution, whose semiclassical interpretation was pointed out by Berger. ${ }^{6}$ However, it is not trivial to correctly account for such contributions in the semiclassical procedure, making a connection to the microscopic approach very desirable.

The early theories of the AHE involved complex calculations with results that were not easy to interpret and often contradicting each other. ${ }^{7}$ The adversity facing these theories stems from the origin of the AHE: It appears due to the interband coherence and not just due to simple changes in the occupation of Bloch states, as was recognized in the early works of Luttinger and Kohn. ${ }^{8,9}$ Nowadays, most treatments of the AHE use either the semiclassical Boltzmann transport theory or the diagrammatic approach based on the KuboStreda linear-response formalism. The equivalence of these two methods for the two-dimensional Dirac-band graphene system has recently been shown by Sinitsyn et al. ${ }^{10}$ who explicitly identified various diagrams of the more systematic Kubo-Streda treatment with the physically more transparent terms of the semiclassical Boltzmann approach.

It is therefore important to also obtain a similarly cohesive understanding of the AHE in other systems such as the two-dimensional (2D) spin-polarized electron gas with Rashba spin-orbit interaction in the presence of pointlike potential impurities, where a series of previous studies has led to a multitude of results with discrepancies arising from the focus on different limits and/or subtle missteps in the calculations. ${ }^{11-17}$ It is the purpose of this paper to review and analyze the previous attempts and to provide a detailed analysis of all contributions to the AHE in a two-dimensional electron gas. Since we have already demonstrated the 
equivalence of the Kubo-Streda formalism and the semiclassical Boltzmann approach with respect to skew scattering in the two-dimensional electron gas in a previous paper, ${ }^{18}$ we will focus here exclusively on the diagrammatic formalism based on the Kubo-Streda treatment.

The outline of this paper is as follows. We start by reviewing and commenting on previous studies of the AHE in the two-dimensional electron gas in Sec. II, where we compare them with our results and discuss the discrepancies and their possible origins. In Sec. III, we present details of our calculation within the diagrammatic Kubo-Streda formalism. In Sec. III C, we provide simple analytical limits of all terms of the anomalous Hall conductivity and discuss the full evaluation in Sec. III D. Finally, in Sec. IV, we present our conclusions.

\section{COMPARISON WITH PREVIOUS APPROACHES}

Currently, there are several publications on the AHE in two-dimensional systems reaching different quantitative predictions even in the same limits. ${ }^{10-17}$ In the present paper, we present a calculation with conclusions that are in disagreement with some previous studies. On such a background, we believe that previous articles have to be discussed in some detail. Below, we review the history of the problem and explain why we think the subject has to be reconsidered.

A first study of the AHE in two-dimensional systems was done by Culcer et al., ${ }^{11}$ who calculated only the intrinsic contribution to the Hall conductivity for a wide class of twodimensional systems, including the Rashba two-dimensional electron gas as a special case. The intrinsic contribution plays a special role in the theory of the AHE because it is not related to the scattering of electrons but is rather caused by the unusual trajectories of electrons under the action of the electric field. However, the disorder contributions can also be important, and further insight was needed in the quest for a quantitatively rigorous theory of the dc-AHE.

The first attempts to understand the disorder effects were done independently by two groups, ${ }^{12,13}$ each employing different approaches. Dugaev et al. ${ }^{12}$ used the version of the Kubo formula, which expresses the Hall conductivity in terms of the causal Green's functions. The intrinsic contribution appears as a result of calculations with bare Green's functions, while disorder effects renormalize the quasiparticle lifetime and the current vertex. This approach is formally rigorous and is similar to the one we adopt in our work. However, our final results are quantitatively different from those found in Ref. 12 due to a subtlety in the calculation of the vertex at the Fermi surface, which was later corrected in the appendix of Ref. 10. Starting with the equation for the renormalized vertex $T_{x}=a k_{x}+b \sigma_{x}+c \sigma_{y}$ and with the assumption that the density of impurities is low, they find correctly that $b=0$ to leading order in $n_{i}$, i.e., $b / a \propto n_{i}$. However, such a term gets multiplied by an equivalent divergent term within the Kubo formula leading to a nonzero contribution to the AHE conductivity to zeroth order in $n_{i}$. In addition, the breakup of the conductivity into $\sigma_{y x}^{\mathrm{I}}$ and $\sigma_{y x}^{\mathrm{II}}$ introduced by Streda $^{20}$ is not strictly followed by Dugaev et al. ${ }^{12}$ who define, instead, $\sigma_{x y}^{\text {II }}$ to be all the terms that arrive from the integration of the Fermi sea (which corresponds to the clean limit Hall conductivity) and terms evaluated at the Fermi surface. As we show here and was also shown in the study of the Dirac Hamiltonian, ${ }^{10} \sigma_{y x}^{\text {II }}$ can be evaluated directly or as a subtraction of the clean limit $\sigma_{y x}$ and $\sigma_{y x}^{\mathrm{I}}$, leading to the correct result when both subbands are occupied.

In contrast to the previous quantum mechanical approach, Sinitsyn et al. ${ }^{13}$ employed the semiclassical wave-packet approach focusing only on the understanding of the side-jump contribution and formulating the semiclassical problem in a gauge invariant form. This work ${ }^{13}$ intentionally avoids a discussion of the skew-scattering contribution due to the asymmetry of the collision term kernel, which is also an important mechanism of the Hall current and can even be parametrically similar to all other contributions, ${ }^{10}$ in the case of Gaussian correlations. Therefore, the work in Ref. 13 is meant as an intuitive introduction into the physics of the anomalous velocity and the side-jump effect but does not offer a rigorous quantitative comparison even in the considered limit of smooth disorder potential.

Subsequently, two papers by Liu et al. ${ }^{14,15}$ studied the problem using the Keldysh technique for linear transport. The Keldysh technique leads to the quantum Boltzmann equation for the diagonal elements of the density matrix in momentum space when only elastic scattering events are considered. In the steady state limit of a weak electric field, this equation can be written as follows:

$$
e \mathbf{E} \cdot \nabla_{\mathbf{p}} \hat{\rho}(\mathbf{p})+i\left[\hat{H}_{0}, \hat{\rho}(\mathbf{p})\right]=\hat{I}_{c o l}(\hat{\rho}(\mathbf{p})),
$$

where $\hat{I}_{c o l}$ contains all disorder dependent terms that become zero when $\hat{\rho}(\mathbf{p})$ is the density matrix in thermodynamic equilibrium and $\hat{H}_{0}$ is the disorder free part of the Hamiltonian. The "hat" means that $\hat{\rho}$ and $\hat{I}_{c o l}$ are matrices in the band index space. The term containing the electric field is called the driving term. In the linear-response approximation, it only depends on the equilibrium part of the density matrix.

To start with, Eq. (1) is correct and is also the starting point of the pioneering work by Luttinger, ${ }^{9}$ and therefore one can compare it directly with steps taken by Liu et al. ${ }^{14,15}$ Luttinger's approach was to split the density matrix into equilibrium and nonequilibrium parts $\hat{\rho}=\hat{\rho}_{e q}+\hat{\rho}_{\text {neq }}$, where $\hat{\rho}_{n e q}$ is linear in electric field. It is this part of the density matrix that is responsible for nonzero currents. For weak disorder potential $\hat{V}$, Luttinger looked for $\hat{\rho}_{\text {neq }}$ as a series in powers of the disorder potential. He found that this series starts from the term of the order $\hat{V}^{-2}$,

$$
\hat{\rho}_{\text {neq }}=\hat{\rho}_{\text {neq }}^{(-2)}+\hat{\rho}_{\text {neq }}^{(-1)}+\hat{\rho}_{\text {neq }}^{(0)}+\cdots .
$$

As pointed out by Luttinger, the leading order term $\hat{\rho}_{\text {neq }}^{(-2)}$ does not contribute to the Hall effect and is only responsible for the longitudinal diffusive current. The term $\hat{\rho}_{\text {neq }}^{(-1)}$ was identified with skew scattering. This term, however, is parametrically very distinct and vanishes in the approximation of purely Gaussian correlations of disorder Fourier components; therefore, Luttinger went to next order and calculated the term $\hat{\rho}_{n e q}^{(0)}$. He found a number of contributions, whose physical meaning he did not clarify. The main conclusion 
was that at this order, both the diagonal and off-diagonal parts of the density matrix become nonzero and contribute to the Hall conductivity, which becomes formally independent on the strength of disorder $\hat{V}$ in the dc limit, although disorder has to be included in the intermediate calculations.

Comparing this with the first work of Liu and Lei, ${ }^{14}$ we find that they determined self-consistently only the offdiagonal part of the density matrix in band index. This is, however, not enough for a rigorous quantitative result because the diagonal part of the $\hat{\rho}_{\text {neq }}^{(0)}$ contribution has been known to be important since Luttinger's pioneering work.

In their next effort, Liu et al. ${ }^{15}$ studied the problem of 2D Rashba systems in small gap semiconductor materials, in which a projection to the conduction band leads to extrinsic type spin-dependent contributions. In this work, they noticed that the diagonal part is important and calculated it numerically. For the driving term in Eq. (1), Liu et al. assume that $\hat{\rho}_{e q}$ is just a diagonal equilibrium Fermi distribution. This would be correct if one was using the basis of the eigenstates of the full Hamiltonian with impurities. However, both Liu et $a l$. and Luttinger work in the chiral basis of the disorder free Hamiltonian $\hat{H}_{0}$. In this basis, the equilibrium state density matrix is no longer diagonal and can also be written as a series in powers of the disorder potential:

$$
\hat{\rho}_{e q}=\hat{\rho}_{e q}^{(0)}+\hat{\rho}_{e q}^{(2)}+\cdots .
$$

Luttinger has shown that in order to properly evaluate the nonequilibrium part $\hat{\rho}_{\text {neq }}^{(0)}$, one should include the second term $\hat{\rho}_{e q}^{(2)}$ of the expansion of the equilibrium density matrix in Eq. (3) into the driving term of Eq. (1). This was not done in Ref. 15 , and therefore we believe that their work is incomplete due to such omission. We also note that the correction of order $\hat{V}^{2}$ in Eq. (3) leads to the Hall current contribution, which was identified in the semiclassical approach ${ }^{19}$ as the anomalous distribution correction and, if omitted, leads to errors of factors of 2 in the typical side-jump-type contributions. ${ }^{7}$ In the Kubo formula approach, neglecting this correction would be equivalent to the unjustified omission of an important subset of Feynman diagrams. ${ }^{10}$ Within the calculation presented here, all these terms are present.

Inoue et al. ${ }^{16}$ calculated the AHE contribution using the same approach we use focusing on the limit of both subbands being occupied and, in addition to the disorder that we consider, incorporating magnetic impurities in the model Hamiltonian. They found that for paramagnetic impurities, the Hall conductivity vanishes. Our more general calculations confirm this result. However, we point to one important difference in its derivation. In both cases, the dc-limit Kubo formula, where the conductivity is expressed via retarded and advanced Green's functions, has been employed to calculate the Hall conductivity. As was shown by Streda, ${ }^{20}$ this version of the Kubo formula contains two parts: $\sigma_{x y}^{\mathrm{I}}$ a contribution from the Fermi surface and $\sigma_{x y}^{\mathrm{II}}$ a contribution from all states of the Fermi sea. The latter part is less known because it does not appear in the expression for the longitudinal conductivity. Inoue et al. ${ }^{16}$ calculated only $\sigma_{x y}^{\mathrm{I}}$, and indeed we find that for their choice of parameters the second part of the conductivity $\sigma_{x y}^{\mathrm{II}}$ vanishes, explaining the agree- ment with our results. In a more general analysis, beyond the limit of weak spin-orbit and Zeeman couplings, we find a nonvanishing $\sigma_{x y}^{\text {II }}$. Our work provides the missing estimate of $\sigma_{x y}^{\text {II }}$ and extends the calculations of Inoue et al. ${ }^{16}$

Finally, the latest work on the subject is by Onoda et al. ${ }^{17}$ The authors used the Keldysh technique, which they reformulated in a way appropriate for multiband problems in a gauge invariant formalism. They also derived a selfconsistent equation, which is the analog of the standard quantum Boltzmann equation, and solved it numerically. Unfortunately, lacking a full understanding of the details of the numerical procedure and the starting equations being very formal within a nonchiral basis, a detailed discussion of their approach cannot be performed here. However, being devoted to the same model, the final results can be compared directly with the possible discrepancies arising from the different limits considered in the disorder distributions in which $n_{i}$ and the disorder strength are two independent parameters in their calculations. Onoda et al. ${ }^{17}$ find a strong skew-scattering contribution of the order of $\epsilon_{S O} V_{i m p} \sigma_{x x} / W^{2}$, where $W$ is the inverse density of states. The skew-scattering term changes sign at the point where the minority band becomes depleted, which they call the resonance point. The authors find also that the side-jump contribution is small in comparison with the intrinsic one. Our results confirm neither of those predictions. We find that for the Rashba model with randomly placed delta-function impurities, the leading part of the skew scattering vanishes identically when the Fermi level is above this resonance point. Although skew scattering could still appear in higher order terms of the Born series, we expect these contributions to be small because they are of higher order in $V_{\text {imp. }}$. On the other hand, Onoda et al. ${ }^{17}$ consider the limit of dilute impurities $n_{i} \rightarrow 0$ independent of the disorder strength $V_{i m p}$ which might be the origin for the discrepancies. Using the Keldysh formalism in the disorder free basis, we have been able to verify analytically our results. Further numerical analysis ${ }^{21}$ of different limits will be necessary to settle the discrepancies with the results by Onoda et al. ${ }^{17}$

\section{ANOMALOUS HALL CONDUCTIVITY OF THE TWO-DIMENSIONAL ELECTRON GAS}

\section{A. Model Hamiltonian}

We consider a spin-polarized two-dimensional electron gas with Rashba spin-orbit interaction

$$
H=\frac{k^{2}}{2 m} \sigma_{0}+\alpha\left(\sigma_{x} k_{y}-\sigma_{y} k_{x}\right)-h \sigma_{z}+V(\mathbf{r}) \sigma_{0},
$$

where $m$ is the effective in-plane mass of the quasiparticles, $\alpha$ the spin-orbit coupling parameter, $h$ the exchange field, and $\sigma_{i}$ the $2 \times 2$ Pauli matrices. The eigenenergies of the clean system are

$$
E_{k \pm}=\frac{k^{2}}{2 m} \pm \lambda_{k} \quad \text { with } \lambda_{k}=\sqrt{h^{2}+\alpha^{2} k^{2}}
$$

and are shown in Fig. 1. The retarded Green's function of the clean system is 

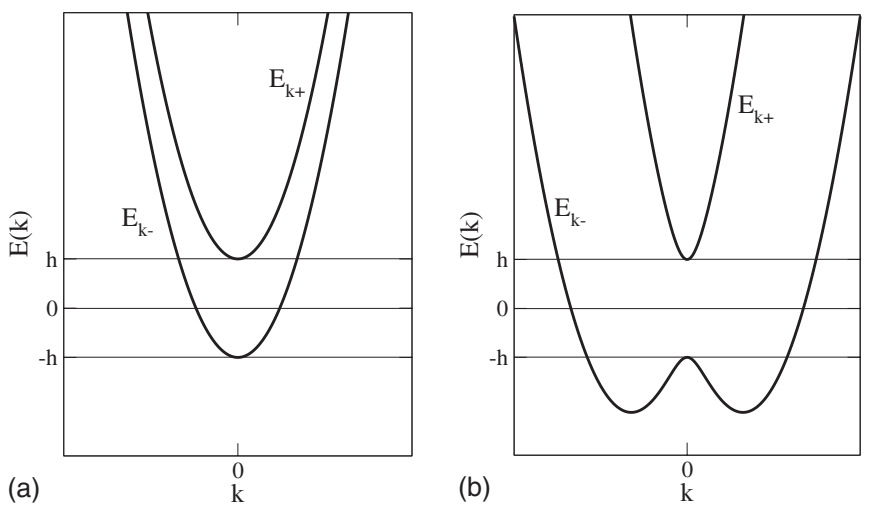

FIG. 1. Single particle dispersion for (a) small spin-orbit interaction $\alpha k_{F} / h=0.2$ and (b) large spin-orbit interaction $\alpha k_{F} / h=2.0$.

$$
\begin{aligned}
G^{(0) R} & =\frac{\left(\omega-\frac{k^{2}}{2 m}+i 0^{+}\right) \sigma_{0}+\alpha k_{y} \sigma_{x}-\alpha k_{x} \sigma_{y}-h \sigma_{z}}{\left(\omega-\frac{k^{2}}{2 m}+i 0^{+}\right)^{2}-h^{2}-\alpha^{2} k^{2}} \\
& =G_{0}^{(0) R} \sigma_{0}+G_{x}^{(0) R} \sigma_{x}+G_{y}^{(0) R} \sigma_{y}+G_{z}^{(0) R} \sigma_{z},
\end{aligned}
$$

with

$$
\begin{gathered}
G_{0}^{(0) R}=\frac{1}{2}\left(G_{+}^{(0)}+G_{-}^{(0)}\right), \quad G_{z}^{(0) R}=-\frac{1}{2} \frac{h}{\lambda_{k}}\left(G_{+}^{(0)}-G_{-}^{(0)}\right), \\
G_{x}^{(0) R}=\frac{1}{2} \frac{\alpha k_{y}}{\lambda_{k}}\left(G_{+}^{(0)}-G_{-}^{(0)}\right), \quad G_{y}^{(0) R}=-\frac{1}{2} \frac{\alpha k_{x}}{\lambda_{k}}\left(G_{+}^{(0)}-G_{-}^{(0)}\right),
\end{gathered}
$$

and

$$
G_{ \pm}^{(0)}=\frac{1}{\omega-E_{k \pm}+i 0^{+}} .
$$

The disorder potential $V(\mathbf{r})$ in Eq. (4) is assumed as spin independent. We consider the model of randomly located $\delta$-function scatterers: $V(\mathbf{r})=\sum_{i} V_{i} \delta\left(\mathbf{r}-\mathbf{R}_{i}\right)$, with random and strength distributions satisfying $\left\langle V_{i}\right\rangle_{d i s}=0,\left\langle V_{i}^{2}\right\rangle_{d i s}=V_{0}^{2} \neq 0$, and $\left\langle V_{i}^{3}\right\rangle_{d i s}=V_{1}^{3} \neq 0$. This model is different from the standard white noise disorder model in which only the second order cumulant is nonzero; $\left\langle\left|V_{\mathbf{k}^{\prime} \mathbf{k}}^{0}\right|^{2}\right\rangle_{\text {dis }}=n_{i} V_{0}^{2}$, where $n_{i}$ is the impurity concentration and other correlators are either zero or related to this correlator by Wick's theorem. The deviation from white noise in our model is quantified by $V_{1} \neq 0$ and is necessary to capture part of the skew-scattering contribution to the anomalous Hall effect.

We calculate the self-energy using the Born approximation:

$$
\begin{aligned}
\Sigma^{R} & =-i\left(\Gamma \sigma_{0}+\Gamma_{z} \sigma_{z}\right) \\
& =-\frac{i}{4} n_{i} V_{0}^{2}\left[\left(\nu_{+}+\nu_{-}\right) \sigma_{0}-h\left(\frac{\nu_{+}}{\lambda_{+}}-\frac{\nu_{-}}{\lambda_{-}}\right) \sigma_{z}\right]
\end{aligned}
$$

where $\nu_{ \pm}$is related to the density of states at the Fermi levels of the two subbands,

$$
\nu_{ \pm}=k\left|\frac{d E_{k \pm}}{d k}\right|^{-1}=\frac{m \lambda_{ \pm}}{\sqrt{\lambda_{F}^{2}+\left(\alpha^{2} m\right)^{2}}},
$$

with

$$
\lambda_{ \pm}=\sqrt{h^{2}+\alpha^{2} k_{ \pm}^{2}}=\sqrt{\lambda_{F}^{2}+\left(\alpha^{2} m\right)^{2}} \mp \alpha^{2} m,
$$

where $\lambda_{F}=\sqrt{h^{2}+2 \alpha^{2} m \epsilon_{F}}$ and

$$
k_{ \pm}=\sqrt{2 m\left[\epsilon_{F}+\alpha^{2} m \mp \sqrt{\lambda_{F}^{2}+\left(\alpha^{2} m\right)^{2}}\right]}
$$

are the Fermi momenta of the two subbands.

Including the self-energy, the impurity averaged Green's function becomes

$$
\begin{aligned}
G^{R} & =\frac{\left(\omega-\frac{k^{2}}{2 m}+i \Gamma\right) \sigma_{0}+\alpha k_{y} \sigma_{x}-\alpha k_{x} \sigma_{y}-\left(h+i \Gamma_{z}\right) \sigma_{z}}{\left(\omega-\frac{k^{2}}{2 m}+i \Gamma\right)^{2}-\left(h+i \Gamma_{z}\right)^{2}-\alpha^{2} k^{2}} \\
& =G_{0}^{R} \sigma_{0}+G_{x}^{R} \sigma_{x}+G_{y}^{R} \sigma_{y}+G_{z}^{R} \sigma_{z} .
\end{aligned}
$$

By comparing this expression with Eq. (6), one observes that the impurity averaged Green's function can be obtained from the Green's function of the clean system by the following replacements:

$$
\omega \rightarrow \omega+i \Gamma, \quad h \rightarrow h+i \Gamma_{z} .
$$

In the limit of small $\Gamma_{z}$, one can therefore expand

$$
\lambda_{k} \rightarrow \sqrt{\left(h+i \Gamma_{z}\right)^{2}+\alpha^{2} k^{2}} \approx \lambda_{k}\left(1+i \frac{h \Gamma_{z}}{\lambda_{k}^{2}}\right) .
$$

Using this approximation, the impurity averaged Green's function can also be written as

$$
\begin{gathered}
G_{0}^{R}=\frac{1}{2}\left(G_{+}^{R}+G_{-}^{R}\right), \\
G_{x}^{R}=\sin \phi \widetilde{G}_{x}^{R}=\frac{1}{2} \frac{\alpha k_{y} \lambda_{k}}{\lambda_{k}^{2}+i \Gamma_{z} h}\left(G_{+}^{R}-G_{-}^{R}\right), \\
G_{y}^{R}=\cos \phi \widetilde{G}_{y}^{R}=-\frac{1}{2} \frac{\alpha k_{x} \lambda_{k}}{\lambda_{k}^{2}+i \Gamma_{z} h}\left(G_{+}^{R}-G_{-}^{R}\right), \\
G_{z}^{R}=-\frac{1}{2} \frac{\lambda_{k}\left(h+i \Gamma_{z}\right)}{\lambda_{k}^{2}+i \Gamma_{z} h}\left(G_{+}^{R}-G_{-}^{R}\right),
\end{gathered}
$$

with

$$
G_{ \pm}^{R}=\frac{1}{\omega-E_{k \pm}+i \Gamma_{ \pm}}
$$

and

$$
\Gamma_{ \pm}=\Gamma \mp \Gamma_{z} \frac{h}{\lambda_{ \pm}} \text {. }
$$

\section{B. General expression for the anomalous Hall conductivity}

According to the Kubo-Streda formalism, ${ }^{20}$ the offdiagonal conductivity can be written as 


$$
\sigma_{y x}=\sigma_{y x}^{\mathrm{I}(a)}+\sigma_{y x}^{\mathrm{I}(b)}+\sigma_{y x}^{\mathrm{II}},
$$

where

$$
\begin{gathered}
\sigma_{y x}^{\mathrm{I}(a)}=\frac{e^{2}}{2 \pi V} \operatorname{Tr}\left\langle v_{y} G^{R}\left(\epsilon_{F}\right) v_{x} G^{A}\left(\epsilon_{F}\right)\right\rangle \\
\sigma_{y x}^{\mathrm{I}(b)}=-\frac{e^{2}}{4 \pi V} \operatorname{Tr}\left\langle v_{y} G^{R}\left(\epsilon_{F}\right) v_{x} G^{R}\left(\epsilon_{F}\right)+v_{y} G^{A}\left(\epsilon_{F}\right) v_{x} G^{A}\left(\epsilon_{F}\right)\right\rangle \\
\sigma_{y x}^{\mathrm{II}}=\frac{e^{2}}{4 \pi V} \int_{-\infty}^{\infty} d \epsilon f(\epsilon) \operatorname{Tr}\left\langle v_{y} G^{R}(\epsilon) v_{x} \frac{\partial G^{R}(\epsilon)}{\partial \epsilon}\right. \\
-v_{y} \frac{\partial G^{R}(\epsilon)}{\partial \epsilon} v_{x} G^{R}(\epsilon)-v_{y} G^{A}(\epsilon) v_{x} \frac{\partial G^{A}(\epsilon)}{\partial \epsilon} \\
\left.+v_{y} \frac{\partial G^{A}(\epsilon)}{\partial \epsilon} v_{x} G^{A}(\epsilon)\right\rangle
\end{gathered}
$$

Here, $\sigma^{\mathrm{I}}$ results from the electrons at the Fermi surface, whereas $\sigma^{\mathrm{II}}$ denotes the contribution of all states of the Fermi sea. For $\sigma^{\mathrm{I}(b)}$ and $\sigma^{\mathrm{II}}$, it is sufficient to calculate the bare bubble contribution in the weak scattering limit ${ }^{10}$ because vertex corrections are of higher order in the scattering rate $\Gamma$. Plugging in the Green's function of Eq. (16) and using the velocity vertices

$$
v_{x}=\frac{k_{x}}{m} \sigma_{0}-\alpha \sigma_{y}, \quad v_{y}=\frac{k_{y}}{m} \sigma_{0}+\alpha \sigma_{x},
$$

one finds that $\sigma^{\mathrm{I}(b)}$ vanishes,

$$
\begin{aligned}
\sigma_{y x}^{\mathrm{I}(b)}= & -\frac{e^{2}}{4 \pi} \frac{1}{(2 \pi)^{2}} \int d^{2} k\left(-i \alpha^{2} G_{0}^{R} G_{z}^{R}+i \alpha^{2} G_{z}^{R} G_{0}^{R}\right. \\
& \left.-i \alpha^{2} G_{0}^{A} G_{z}^{A}+i \alpha^{2} G_{z}^{A} G_{0}^{A}\right)=0 .
\end{aligned}
$$

The bare contribution of $\sigma^{\mathrm{II}}$ in the clean limit, i.e., for $\Gamma_{+}$ $=\Gamma_{-}=0^{+}$, can be calculated by integration (see Appendix A) and yields

$$
\sigma_{y x}^{\mathrm{II}}=-\frac{e^{2}}{4 \pi}\left(1-\frac{h}{\sqrt{h^{2}+2 \alpha^{2} m \epsilon_{F}+\left(\alpha^{2} m\right)^{2}}}\right) \Theta\left(h-\epsilon_{F}\right),
$$

where $\partial G_{ \pm}^{R / A} / \partial \epsilon=-\left(G_{ \pm}^{R / A}\right)^{2}$ has been used. Including the real scattering rates $\Gamma_{+}$and $\Gamma_{-}$does not lead to qualitatively different results but mainly causes a slight smearing. Thus, we consider it as sufficient to focus on the clean limit contribution of $\sigma^{\mathrm{II}}$.

For $\sigma^{\mathrm{I}(a)}$, vertex corrections can be of similar magnitude as the bare bubble and thus have to be considered carefully. In the weak scattering limit, contributions of higher order impurity scattering vertices are small leaving only laddertype vertex corrections and the $V_{1}^{3} /\left(n_{i} V_{0}^{4}\right)$ skew-scattering contribution as the important terms. ${ }^{18}$ Thus, we decompose $\sigma^{\mathrm{I}(a)}$ in the following way:

$$
\sigma_{y x}^{\mathrm{I}(a)}=\sigma_{y x}^{\mathrm{I}(a), b}+\sigma_{y x}^{\mathrm{I}(a), l}+\sigma_{y x}^{\mathrm{I}(a), s},
$$

where $\sigma_{y x}^{\mathrm{I}(a), b}$ is the bare bubble contribution [Fig. 2(a)], $\sigma_{y x}^{\mathrm{I}(a), l}$ the ladder vertex corrections [Fig. 2(b)], and $\sigma_{y x}^{\mathrm{I}(a), s}$ the skew-
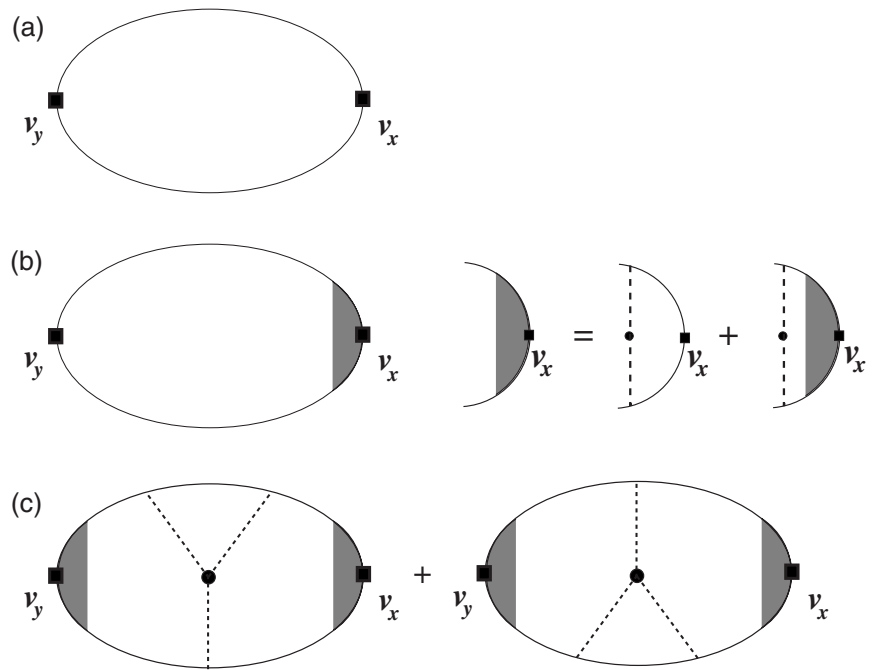

FIG. 2. Diagrammatic representation (a) of the bare bubble, (b) of the ladder vertex corrections, and (c) of the skew-scattering contribution.

scattering contribution [Fig. 2(c)]. With respect to the skewscattering contribution, we have shown ${ }^{18}$ that only the diagrams with a single third order vertex [see Fig. 2(c)] contribute to order $V_{1}^{3} /\left(n_{i} V_{0}^{4}\right)$. In this diagram, both vertices have to be renormalized by ladder vertex corrections.

\section{Bare bubble}

The calculation of the bare bubble contribution proceeds as follows:

$$
\begin{aligned}
\sigma_{y x}^{\mathrm{I}(a), b} & =\frac{e^{2}}{2 \pi} \iint \frac{d k k d \phi}{(2 \pi)^{2}} \operatorname{Tr}\left[v_{y} G^{R}\left(\epsilon_{F}\right) v_{x} G^{A}\left(\epsilon_{F}\right)\right] \\
& =2 i \alpha \int \frac{d k k}{2 \pi}\left(\frac{k}{m}\left(\widetilde{G}_{y}^{R} G_{z}^{A}-G_{z}^{R} \widetilde{G}_{y}^{A}\right)-\alpha\left(G_{0}^{R} G_{z}^{A}-G_{z}^{R} G_{0}^{A}\right)\right) \\
& =2 i \alpha\left(2 I_{3}-\alpha I_{2}\right),
\end{aligned}
$$

where (for explicit evaluation of integrals $I_{1}, I_{2}, I_{3}$, and $I_{4}$, see Appendix B)

$$
\begin{gathered}
I_{1}=\frac{1}{2 \pi} \int d k k\left(G_{0}^{R} G_{0}^{A}-G_{z}^{R} G_{z}^{A}\right) \\
\approx \frac{1}{8}\left[\left(1-\frac{h^{2}}{\lambda_{+}^{2}}\right) \frac{\nu_{+}}{\Gamma_{+}}+\left(1-\frac{h^{2}}{\lambda_{-}^{2}}\right) \frac{\nu_{-}}{\Gamma_{-}}\right], \\
I_{2}=\frac{1}{2 \pi} \int d k k\left(G_{0}^{R} G_{z}^{A}-G_{z}^{R} G_{0}^{A}\right) \\
\approx-\frac{i}{4}\left(\frac{\nu_{+} h}{\lambda_{+}^{2}}+\frac{\nu_{-} h}{\lambda_{-}^{2}}-\frac{\Gamma_{z}}{\Gamma_{+}} \frac{\nu_{+} \alpha^{2} k_{+}^{2}}{\lambda_{+}^{3}}+\frac{\Gamma_{z}}{\Gamma_{-}} \frac{\nu_{-} \alpha^{2} k_{-}^{2}}{\lambda_{-}^{3}}\right), \\
I_{3}=\frac{1}{2 \pi} \int d k \frac{k^{2}}{2 m}\left(\widetilde{G}_{y}^{R} G_{z}^{A}-G_{z}^{R} \widetilde{G}_{y}^{A}\right) \\
\approx-\frac{i}{4} \alpha \Gamma_{z}\left[\frac{\nu_{+}}{\Gamma_{+} \lambda_{+}}\left(\frac{\epsilon_{F}}{\lambda_{+}}-1\right)+\frac{\nu_{-}}{\Gamma_{-} \lambda_{-}}\left(\frac{\epsilon_{F}}{\lambda_{-}}+1\right)\right]
\end{gathered}
$$




$$
\begin{aligned}
I_{4} & =\frac{1}{2 \pi} \int d k \frac{k^{2}}{2 m}\left(G_{0}^{R} \widetilde{G}_{y}^{A}+\widetilde{G}_{y}^{R} G_{0}^{A}\right) \\
& \approx-\frac{1}{4} \alpha\left[\epsilon_{F}\left(\frac{\nu_{+}}{\Gamma_{+} \lambda_{+}}-\frac{\nu_{-}}{\Gamma_{-} \lambda_{-}}\right)-\left(\frac{\nu_{+}}{\Gamma_{+}}+\frac{\nu_{-}}{\Gamma_{-}}\right)\right] .
\end{aligned}
$$

\section{Ladder diagrams}

For the ladder terms $\sigma_{y x}^{\mathrm{I}(a), l}$, we sum the vertex corrections in front of the $v_{x}$ vertex as indicated in Fig. 2(b). Starting from the momentum integrated bare velocity vertex

$$
\iint \frac{d k k d \phi}{(2 \pi)^{2}} G^{R}\left(\epsilon_{F}\right) v_{x} G^{A}\left(\epsilon_{F}\right)=\gamma_{x} \sigma_{x}+\gamma_{y} \sigma_{y},
$$

with

$$
\gamma_{x}=i\left(I_{3}-\alpha I_{2}\right), \quad \gamma_{y}=I_{4}-\alpha I_{1},
$$

one finds for the renormalized vertex

$$
\begin{aligned}
\Gamma_{v_{x}}= & \Gamma_{x} \sigma_{x}+\Gamma_{y} \sigma_{y} \\
= & \gamma_{x} \sigma_{x}+\gamma_{y} \sigma_{y} \\
& +n_{i} V_{0}^{2} \iint \frac{d k k d \phi}{(2 \pi)^{2}} G^{R}\left(\epsilon_{F}\right)\left(\gamma_{x} \sigma_{x}+\gamma_{y} \sigma_{y}\right) G^{A}\left(\epsilon_{F}\right) \\
= & \gamma_{x} \sigma_{x}+\gamma_{y} \sigma_{y} \\
& +n_{i} V_{0}^{2}\left[\left(I_{1} \Gamma_{x}+i I_{2} \Gamma_{y}\right) \sigma_{x}+\left(I_{1} \Gamma_{y}-i I_{2} \Gamma_{x}\right) \sigma_{y}\right],
\end{aligned}
$$

and thus

$$
\begin{aligned}
\left(\begin{array}{c}
\Gamma_{x} \\
\Gamma_{y}
\end{array}\right)= & \frac{1}{\left(1-n_{i} V_{0}^{2} I_{1}\right)^{2}-\left(n_{i} V_{0}^{2} I_{2}\right)^{2}}\left(\begin{array}{cc}
1-n_{i} V_{0}^{2} I_{1} & i n_{i} V_{0}^{2} I_{2} \\
-i n_{i} V_{0}^{2} I_{2} & 1-n_{i} V_{0}^{2} I_{1}
\end{array}\right) \\
& \times\left(\begin{array}{c}
\gamma_{x} \\
\gamma_{y}
\end{array}\right) .
\end{aligned}
$$

The ladder diagrams are therefore given by

$$
\begin{aligned}
\sigma_{y x}^{\mathrm{I}(a), l} & =\frac{e^{2}}{2 \pi} \iint \frac{d k k d \phi}{(2 \pi)^{2}} \operatorname{Tr}\left[G^{A}\left(\epsilon_{F}\right) v_{y} G^{R}\left(\epsilon_{F}\right)\left(\Gamma_{x} \sigma_{x}+\Gamma_{y} \sigma_{y}\right)\right] \\
& =-\frac{e^{2}}{2 \pi} 2\left(\gamma_{y} \Gamma_{x}+\gamma_{x} \Gamma_{y}\right) \\
& =-\frac{e^{2}}{\pi} \frac{n_{i} V_{0}^{2}\left[2 \gamma_{x} \gamma_{y}\left(1-n_{i} V_{0}^{2} I_{1}\right)+i n_{i} V_{0}^{2} I_{2}\left(\gamma_{y}^{2}-\gamma_{x}^{2}\right)\right]}{\left(1-n_{i} V_{0}^{2} I_{1}\right)^{2}-\left(n_{i} V_{0}^{2} I_{2}\right)^{2}} .
\end{aligned}
$$

In the weak scattering limit, this reduces to

$$
\sigma_{y x}^{\mathrm{I}(a), l}=-\frac{e^{2}}{\pi} \frac{n_{i} V_{0}^{2}\left[2 \gamma_{x} \gamma_{y}\left(1-n_{i} V_{0}^{2} I_{1}\right)+i n_{i} V_{0}^{2} I_{2} \gamma_{y}^{2}\right]}{\left(1-n_{i} V_{0}^{2} I_{1}\right)^{2}} .
$$

\section{Skew scattering}

For skew scattering, we consider only diagrams with a single third order impurity vertex and both external current vertices renormalized by ladder vertex corrections as indicated in Fig. 2(c). In analogy to the renormalized $v_{x}$ vertex in

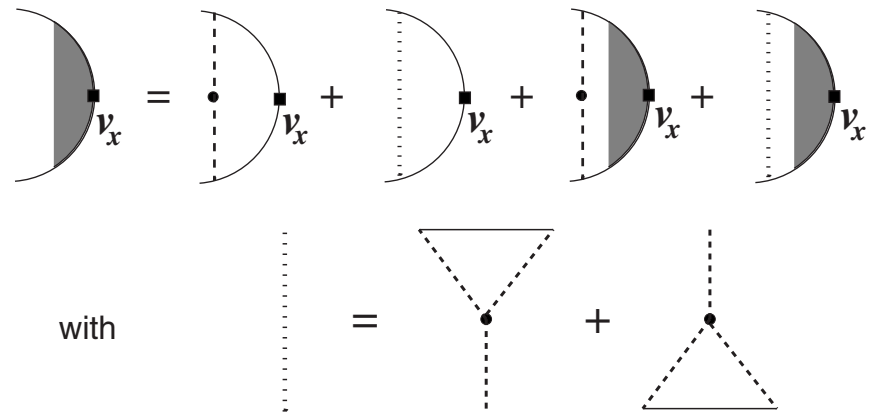

FIG. 3. Full vertex including ladder and skew-scattering diagrams.

Eq. (29), also the renormalized $v_{y}$ vertex can be calculated and expressed via $\Gamma_{x}$ and $\Gamma_{y}$ as

$$
\Gamma_{v_{y}}=-\Gamma_{y} \sigma_{x}-\Gamma_{x} \sigma_{y}
$$

Using these expressions, the skew-scattering diagram of Fig. 2(c) yields

$$
\begin{aligned}
\sigma_{y x}^{\mathrm{I}(a), s}= & \frac{e^{2}}{2 \pi} \frac{n_{i} V_{1}^{3}}{2 \pi} \int d k k \operatorname{Tr}\left[\Gamma_{v_{y}} G^{R}\left(\epsilon_{F}\right) \Gamma_{v_{x}}+\Gamma_{v_{y}} \Gamma_{v_{x}} G^{A}\left(\epsilon_{F}\right)\right] \\
= & \frac{e^{2}}{2 \pi} \frac{i V_{1}^{3}}{V_{0}^{2}} \operatorname{Tr}\left[-\Gamma_{v_{y}}\left(\Gamma \sigma_{0}+\Gamma_{z} \sigma_{z}\right) \Gamma_{v_{x}}\right. \\
& \left.+\Gamma_{v_{y}} \Gamma_{v_{x}}\left(\Gamma \sigma_{0}+\Gamma_{z} \sigma_{z}\right)\right] \\
= & \frac{e^{2}}{2 \pi} \frac{V_{1}^{3}}{V_{0}^{2}} i \Gamma_{z} \operatorname{Tr}\left[( \Gamma _ { y } \sigma _ { x } + \Gamma _ { x } \sigma _ { y } ) \left(\sigma_{z}\left(\Gamma_{x} \sigma_{x}+\Gamma_{y} \sigma_{y}\right)\right.\right. \\
& \left.\left.-\left(\Gamma_{x} \sigma_{x}+\Gamma_{y} \sigma_{y}\right) \sigma_{z}\right)\right] \\
= & \frac{e^{2}}{2 \pi} \frac{V_{1}^{3}}{V_{0}^{2}} 4 \Gamma_{z}\left(\Gamma_{y}^{2}-\Gamma_{x}^{2}\right) .
\end{aligned}
$$

From this expression, it is evident that the skew-scattering contribution vanishes as soon as $\Gamma_{z}=0$, implying that the lifetimes in both bands become equal since $\Gamma_{-}-\Gamma_{+}$ $=\Gamma_{z}\left(h / \lambda_{-}+h / \lambda_{+}\right)$vanishes for $\Gamma_{z}=0$. Plugging in $\Gamma_{x}$ and $\Gamma_{y}$ from Eq. (30), one finds ${ }^{18}$ in the weak scattering limit, i.e., neglecting higher order impurity terms,

$$
\begin{gathered}
\sigma_{y x}^{\mathrm{I}(a), s}=\frac{e^{2}}{2 \pi} \frac{4 V_{1}^{3} \Gamma_{z} \gamma_{y}^{2}}{V_{0}^{2}\left(1-n_{i} V_{0}^{2} I_{1}\right)^{2}} \\
=\frac{e^{2}}{2 \pi} \frac{V_{1}^{3}}{n_{i} V_{0}^{4}} \frac{h \lambda_{-} \alpha^{2} k_{-}^{4}}{\nu_{-}\left(3 h^{2}+\lambda_{-}^{2}\right)^{2}} .
\end{gathered}
$$

It can be shown easily that considering the weak scattering limit of the full vertex shown in Fig. 3 yields exactly the same result as Eq. (35b), i.e., to order $V_{1}^{3} /\left(n_{i} V_{0}^{4}\right)$ it reduces to the elementary skew-scattering diagram depicted in Fig. 2(c).

\section{Simple limits}

\section{Both subbands occupied}

In the situation that both subbands are partially occupied, i.e., $\epsilon_{F}>h$, all contributions to the anomalous Hall conduc- 
tivity vanish. For $\sigma_{y x}^{\mathrm{II}}$, this is immediately evident from Eq. (23). For the skew-scattering contribution, which is proportional to $\Gamma_{z}$ [see Eq. (35b)], one observes easily that $\sigma_{v x}^{\mathrm{I}(a), s}$ $=0$ because $\Gamma_{z}=0$ [see Eq. (9)] due to $\nu_{+} / \lambda_{+}-\nu_{-} / \lambda_{-}=0$ [see Eq. (10)].

With respect to the bare bubble and ladder diagrams, we will show in the following that they cancel mutually. For $\epsilon_{F}>h$, the integrals in Eq. (26) simplify to

$$
I_{1}=\frac{\alpha^{2} m^{2} \epsilon_{F}}{2 \lambda_{F}^{2} \Gamma}, \quad I_{2}=-\frac{i h m}{2 \lambda_{F}^{2}}, \quad I_{3}=0, \quad I_{4}=\frac{\alpha m}{2 \Gamma},
$$

and the bare momentum integrated vertices in Eq. (28) are

$$
n_{i} V_{0}^{2} \gamma_{x}=-\frac{\alpha h \Gamma}{\lambda_{F}^{2}}, \quad n_{i} V_{0}^{2} \gamma_{y}=\alpha\left(1-\frac{\alpha^{2} m \epsilon_{F}}{\lambda_{F}^{2}}\right) .
$$

This gives for the bare bubble in Eq. (25)

$$
\sigma_{y x}^{\mathrm{I}(a), b}=-\frac{e^{2}}{2 \pi} \frac{\alpha^{2} m h}{\lambda_{F}^{2}} .
$$

For the ladder diagrams, we need also

$$
1-n_{i} V_{0}^{2} I_{1}=\frac{n_{i} V_{0}^{2} \gamma_{y}}{\alpha}, \quad-i n_{i} V_{0}^{2} I_{2}=\frac{n_{i} V_{0}^{2} \gamma_{x}}{\alpha},
$$

yielding

$$
\sigma_{y x}^{\mathrm{I}(a), l}=-\frac{e^{2}}{\pi} \frac{\alpha}{n_{i} V_{0}^{2}} \frac{2 \gamma_{x} \gamma_{y}^{2}-\gamma_{x} \gamma_{y}^{2}+\gamma_{x}^{3}}{\gamma_{x}^{2}+\gamma_{y}^{2}}=-\frac{e^{2}}{\pi} \frac{\alpha \gamma_{x}}{n_{i} V_{0}^{2}}=\frac{e^{2}}{2 \pi} \frac{\alpha^{2} m h}{\lambda_{F}^{2}},
$$

and thus

$$
\sigma_{y x}^{\mathrm{I}(a), b}+\sigma_{y x}^{\mathrm{I}(a), l}=0,
$$

i.e., the contributions of the bare bubble and the ladder diagrams cancel mutually.

\section{Only majority band occupied}

In the opposite situation, where only the majority band is partially occupied, we have $\nu_{+}=0$ and therefore $\Gamma_{z} \neq 0$. In this case, all terms contribute to the anomalous Hall conductivity. In the following, we restrict our analysis to Fermi energies $\epsilon_{F}>-h$, i.e., we disregard the region of very small Fermi energies, where the valley structure of the majority band becomes important [see Fig. 1(b)], and discuss the results in two simple limits: (i) small spin-orbit interaction, $\alpha k_{F} \ll h$, and (ii) small magnetization, $h \ll \alpha k_{F}$.

In the limit of small spin-orbit interaction, $\alpha k_{F} \ll h$, the sum of bare bubble and ladder vertex corrections becomes

$$
\sigma_{y x}^{\mathrm{I}(a), b}+\sigma_{y x}^{\mathrm{I}(a), l}=\frac{e^{2}}{2 \pi} \frac{\left(\alpha k_{F}\right)^{2}}{16 h \epsilon_{F}}\left(3 \frac{\epsilon_{F}}{h}+1\right)\left(-\frac{\epsilon_{F}}{h}+1\right),
$$

the contribution from the states of the full Fermi sea is

$$
\sigma_{y x}^{\mathrm{II}}=-\frac{e^{2}}{4 \pi} \frac{\left(\alpha k_{F}\right)^{2}}{2 h^{2}},
$$

and the skew-scattering term is

$$
\sigma_{y x}^{\mathrm{I}(a), s}=\frac{e^{2}}{2 \pi} \frac{\left(\alpha k_{F}\right)^{2}}{8 \epsilon_{F} n_{i} V_{0}} \frac{V_{1}^{3}}{V_{0}^{3}} \frac{\left(\epsilon_{F}+h\right)^{2}}{h^{2}} .
$$

In the opposite limit of small exchange field $h \ll \alpha k_{F}$, considering first a spin-orbit interaction still smaller than the Fermi energy $\alpha k_{F} \ll \epsilon_{F}$, we find for the sum of bare bubble and ladder vertex corrections

$$
\sigma_{y x}^{\mathrm{I}(a), b}+\sigma_{y x}^{\mathrm{I}(a), l}=-\frac{e^{2}}{2 \pi} \frac{3 h \epsilon_{F}}{\left(\alpha k_{F}\right)^{2}},
$$

for the contribution from the states of the full Fermi sea

$$
\sigma_{y x}^{\mathrm{II}}=-\frac{e^{2}}{4 \pi}\left(1-\frac{h}{\alpha k_{F}}\right),
$$

and for the skew-scattering term

$$
\sigma_{y x}^{\mathrm{I}(a), s}=\frac{e^{2}}{2 \pi} \frac{V_{1}^{3}}{V_{0}^{3}} \frac{2 h \epsilon_{F}}{n_{i} V_{0} \alpha k_{F}} .
$$

In the same limit where the exchange field is small, $h$ $\ll \alpha k_{F}$, but the spin-orbit interaction is now larger than the Fermi energy, $\alpha k_{F} \gg \epsilon_{F}$, we find for the sum of bare bubble and ladder vertex corrections

$$
\sigma_{y x}^{\mathrm{I}(a), b}+\sigma_{y x}^{\mathrm{I}(a), l}=-\frac{e^{2}}{2 \pi} \frac{2 h \epsilon_{F}^{3}}{\left(\alpha k_{F}\right)^{4}},
$$

for the contribution from the states of the full Fermi sea

$$
\sigma_{y x}^{\mathrm{II}}=-\frac{e^{2}}{4 \pi}\left(1-\frac{2 h \epsilon_{F}}{\left(\alpha k_{F}\right)^{2}}\right),
$$

and for the skew-scattering term

$$
\sigma_{y x}^{\mathrm{I}(a), s}=\frac{e^{2}}{2 \pi} \frac{V_{1}^{3}}{V_{0}^{3}} \frac{h}{n_{i} V_{0}} .
$$

\section{Discussion}

We now discuss the full evaluation of the anomalous Hall conductivity in the limit of small spin-orbit interaction, $\alpha k_{F}$ $\ll h$, and in the opposite limit of strong spin-orbit interaction, $\alpha k_{F} \gg h, \epsilon_{F}$. For the following discussion, we will express all quantities in terms of the exchange field $h$, which we define as $h=1$. Furthermore, we will set $m=1$, we choose $V_{1}=V_{0}$, and use an impurity concentration of $n_{i}=0.1$.

In Fig. 4, we show the anomalous Hall conductivity for a small spin-orbit interaction of $\alpha k_{F} / h=0.2$ as a function of the Fermi energy $\epsilon_{F} / h$ and the scattering rate $1 / \tau=n_{i} V_{0}^{2} m$ for an impurity concentration of $n_{i}=0.1$. The upper left panel shows the total anomalous Hall conductivity, i.e., the sum of skew scattering (upper right panel), of bare bubble and ladder diagrams (lower left panel) and of the contribution from the whole Fermi sea (lower right panel). Obviously, all contributions to the total conductivity vanish for $\epsilon_{F}>h$, i.e., when both subbands are occupied which agrees with our analysis in Sec. III C 1. Furthermore we observe that not only $\sigma_{y x}^{\mathrm{II}}$ but also the bare bubble and ladder vertex corrections $\sigma_{y x}^{\mathrm{I}(a), b}+\sigma_{y x}^{\mathrm{I}(a), l}$ [see Eq. (42)] are independent of impurity 
$1 / \tau$

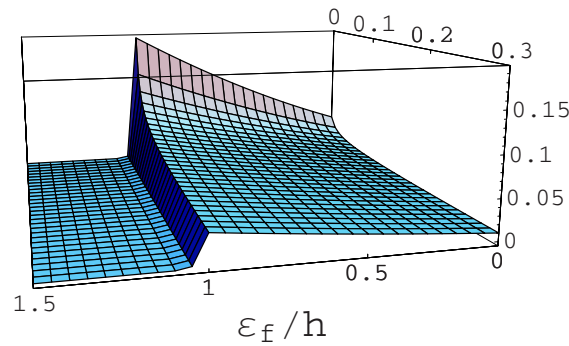

$1 / \tau$

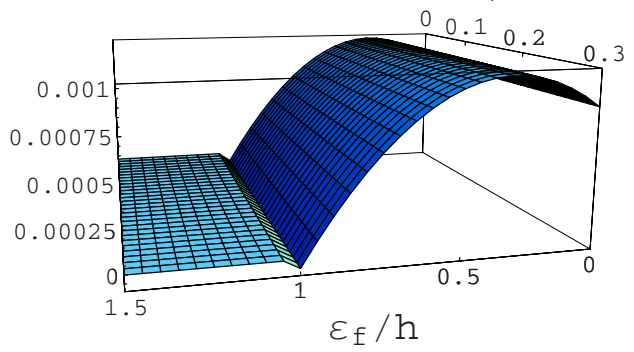

$1 / \tau$

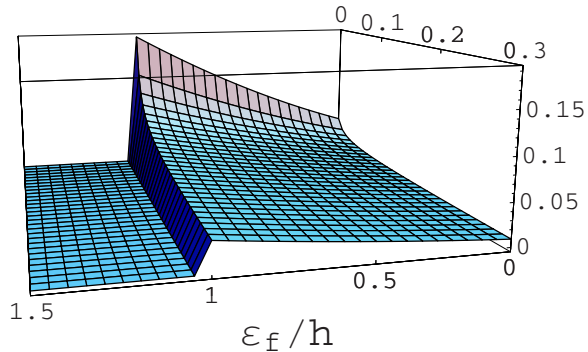

$1 / \tau$

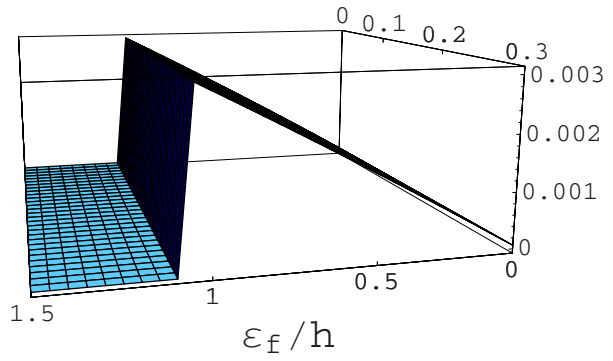

FIG. 4. (Color online) Anomalous Hall conductivity for $\alpha k_{F} / h=0.2$ and an impurity concentration of $n_{i}=0.1$ plotted as a function of $\epsilon_{F} / h$ (from right to left) and as a function of $1 / \tau=n_{i} m V_{0}^{2}$ in units of $h$ (from back to front): upper left panel, total anomalous Hall conductivity [Eq. (19)]; upper right panel, skew-scattering contribution [Eq. (35b)]; lower left panel; bare bubble plus ladder vertex corrections [Eq. (25)+Eq. (31)]; lower right panel, $-\sigma^{\mathrm{II}}$ [Eq. (23)]. All conductivities are plotted in units of $e^{2}$.

scattering. Both contributions are small: $\sigma^{\mathrm{II}}$ contains a small prefactor of $\left(\alpha k_{F} / h\right)^{2}$ [see Eq. (43)] and $\sigma_{y x}^{\mathrm{I}(a), b}+\sigma_{y x}^{\mathrm{I}(a), l}$ a small prefactor of $\left(\alpha k_{F}\right)^{2} /\left(h \epsilon_{F}\right)$ [see Eq. (42)]. The skew-scattering contribution, on the other hand, has a prefactor of $\alpha k_{F} /\left(n_{i} V_{0}\right)$, which diverges for $V_{0} \rightarrow 0$, i.e., $1 / \tau \rightarrow 0$ [see Eq. (44)], and therefore overcompensates the small prefactor of $\alpha k_{F} / \epsilon_{F}\left[\right.$ see Eq. (44)] when the impurity potentials $V_{0}$ become small enough. Thus, for the parameters chosen in Fig. 4, the skew-scattering term outweighs the other contributions by orders of magnitude, and therefore the total anomalous Hall conductivity is almost identical to the skewscattering term. It increases quadratically with $\epsilon_{F} / h$ [see Eq. (44)] and then vanishes suddenly for $\epsilon_{F}>h$.

Figure 5 displays the anomalous Hall conductivity in a
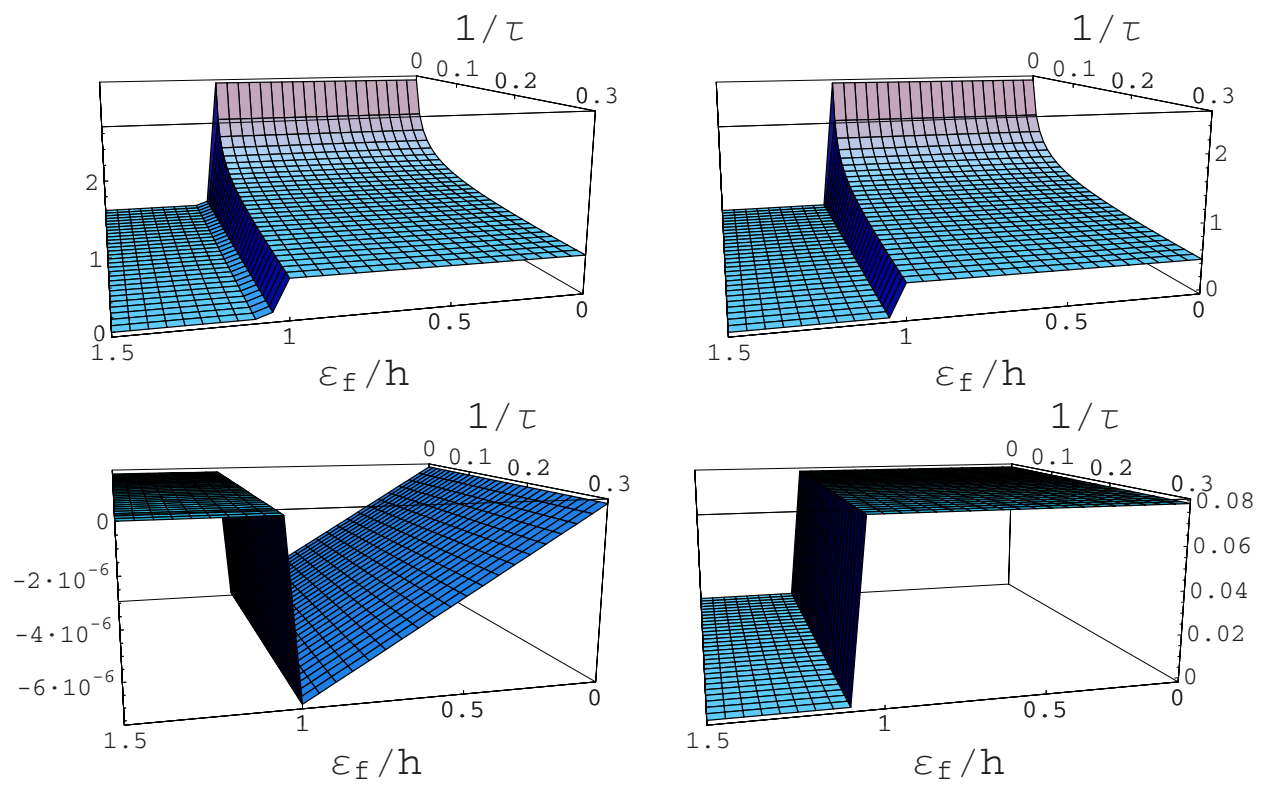

FIG. 5. (Color online) Anomalous Hall conductivity for $\alpha k_{F} / h=10.0$ and an impurity concentration of $n_{i}=0.1$ plotted as a function of $\epsilon_{F} / h$ (from right to left) and as a function of $1 / \tau=n_{i} m V_{0}^{2}$ in units of $h$ (from back to front): upper left panel, total anomalous Hall conductivity [Eq. (19)]; upper right panel, skew-scattering contribution [Eq. (35b)]; lower left panel, bare bubble plus ladder vertex corrections [Eq. (25)+Eq. (31)]; lower right panel, $-\sigma^{\mathrm{II}}$ [Eq. (23)]. All conductivities are plotted in units of $e^{2}$. 
similar way as Fig. 4 only for a large spin-orbit interaction of $\alpha k_{F} / h=10$. Again, $\sigma_{y x}^{\mathrm{I}(a), b}+\sigma_{y x}^{\mathrm{I}(a), l}$ turns out to be independent of the impurity parameters and even smaller in magnitude as before because now it is suppressed by a small prefactor of $\left(h \epsilon_{F}^{3}\right) /\left(\alpha k_{F}\right)^{4}$ [see Eq. (48)]. Analogous to the limit of small spin-orbit interaction, the total anomalous Hall conductivity is dominated by the skew-scattering contribution, which contains no small prefactor and, due to the factor of $h /\left(n_{i} V_{0}\right)$, grows rapidly for small impurity potentials, $V_{0} \rightarrow 0$, i.e., $1 / \tau \rightarrow 0$ [see Eq. (50)]. In the limit of large spin-orbit interaction, $\alpha k_{F} \gg \epsilon_{F}$, the skew scattering and thus the total anomalous Hall conductivity are independent of the Fermi energy $\epsilon_{F}$ for $\epsilon_{F}<h$ [see Eq. (50)] and then abruptly drops to zero for $\epsilon_{F}>h$.

\section{CONCLUSIONS}

In summary, we have investigated the anomalous Hall conductivity in a spin-polarized two-dimensional electron gas with Rashba spin-orbit interaction in the presence of pointlike potential impurities. Our calculations have been performed within diagrammatic perturbation theory based on the Kubo-Streda formula, an approach which has previously been shown to yield equivalent results to the semiclassical Boltzmann treatment. ${ }^{10,18}$

Comparing our results with previous calculations, we have been able to sort out contradictions existing in the literature. We have found that within the model Hamiltonian considered, all contributions to the anomalous Hall conductivity vanish as soon as the minority band becomes partially filled, i.e., as soon as the Fermi energy becomes larger than the internal Zeeman field. For smaller Fermi energies, all contributions are finite with $\sigma_{y x}^{\mathrm{II}}$, the contribution from all states of the Fermi sea, being the smallest term at least in the limits of weak and of strong spin-orbit interaction. The vertex corrections, which represent disorder contributions, can be of similar magnitude as the intrinsic contribution and turn out to be independent of the impurity concentration and impurity potential at least in the limits of small and of strong spin-orbit interaction. In the weak scattering limit, the dominant contribution results from skew scattering because due to its $1 /\left(n_{i} V_{0}\right)$ dependence, it outweighs all other terms. Moreover, the intrinsic and the side-jump terms contain higher orders of small prefactors than the skew-scattering contribution.

\section{ACKNOWLEDGMENTS}

Fruitful discussions with S. Onoda and N. Nagaosa are gratefully acknowledged. This work was supported by SPP 1285 of the DFG, by ONR under Grant No. ONRN000140610122, by the NSF under Grant No. DMR0547875, by SWAN-NRI, by EU Grant No. IST-015728, by EPSRC Grant No. GR/S81407/01, by GACR and AVCR Grants No. 202/05/0575, No. FON/06/E002, No. AV0Z1010052, and No. LC510, by the DOE under Grant No. DE-AC52-06NA25396, and by the University of Kansas General Research Fund Allocation No. 2302015. J.I. thanks the Next Generation Super Computing Project, Nanoscience
Program, MEXT, Japan, and Grant-in-Aid for the 21st Century COE "Frontiers of Computational Science" for financial support. J.S. is a Cottrell Scholar of the Research Foundation. V.K.D. was supported by FCT Grant No. PTDC/FIS/ 70843/2006 in Portugal, Polish Ministry of Science and Higher Education as research projects in years 2006-2009 and 2007-2010, and by STCU Grant No. 3098 in Ukraine.

\section{APPENDIX A: DEFINITION AND INTEGRATION OF $\boldsymbol{\sigma}^{\mathrm{II}}$}

\section{Integration of $\sigma_{y x}^{\mathrm{II}}$ using the theorem of residues}

Here, we discuss and further clarify the meaning of the $\sigma_{y x}^{\mathrm{II}}$ contribution to the anomalous Hall conductivity in Eq. (20).

The definition of the conductivity tensor in the form of a sum, $\sigma_{y x}=\sigma_{y x}^{\mathrm{I}}+\sigma_{y x}^{\mathrm{II}}$, has been proposed long ago by Streda. ${ }^{20}$ The idea of this separation is that the term $\sigma_{y x}^{\mathrm{I}}$ corresponds to the well-known classical Drude-Zener formula of conductivity, whereas $\sigma_{y x}^{\text {II }}$ has no obvious classical analogy. The other important point is that $\sigma_{y x}^{\text {II }}$ does not depend on scattering from impurities. Later on, Streda's separation of the offdiagonal conductivity in two parts has been rederived by using the Kubo formalism. ${ }^{22}$

As we see from Eq. (20), the contribution $\sigma_{y x}^{\mathrm{I}}=\sigma_{y x}^{\mathrm{I}(a)}$ $+\sigma_{y x}^{\mathrm{I}(b)}$ is related only to the states at the Fermi surface, whereas $\sigma_{y x}^{\mathrm{II}}$ formally includes the contribution of all states with energies below the Fermi level. However, in the integral over energy in $\sigma_{y x}^{\mathrm{II}}$, a part with $\varepsilon=\varepsilon_{F}$ can be additionally separated so that we can present $\sigma_{y x}^{\mathrm{II}}$ as $\sigma_{y x}^{\mathrm{II}}=\sigma_{y x}^{(i n t)}-\sigma_{y x}^{\mathrm{I}(i n t)}$, where $\sigma_{y x}^{(i n t)}$ includes integration over all states with $\varepsilon<\varepsilon_{F}$. Mathematically, the reason for this is a singularity at the point $\varepsilon=\varepsilon_{F}$ related to the Fermi function $f(\varepsilon)$ in the limit of zero temperature. It was already discussed in Appendix $\mathrm{C}$ of Ref. 10 in the context of the two-dimensional Dirac model.

Let us demonstrate it now within the model of twodimensional electron gas with Rashba spin-orbit interaction. Starting from $\sigma_{y x}^{\text {II }}$ of Eq. (20) with the Green's functions of a clean crystal [Eq. (6)] and calculating the trace, we can present it as

$$
\begin{aligned}
\sigma_{y x}^{\mathrm{II}}= & -\frac{i e^{2} \alpha^{2} h}{2 \pi} \int \frac{d^{2} k}{(2 \pi)^{2}} \frac{1}{\lambda_{k}} \int_{-\infty}^{\infty} f(\varepsilon)\left(-G_{-}^{(0) R} \frac{\partial G_{+}^{(0) R}}{\partial \varepsilon}\right. \\
& \left.+G_{+}^{(0) R} \frac{\partial G_{-}^{(0) R}}{\partial \varepsilon}+G_{-}^{(0) A} \frac{\partial G_{+}^{(0) A}}{\partial \varepsilon}-G_{+}^{(0) A} \frac{\partial G_{-}^{(0) A}}{\partial \varepsilon}\right) .
\end{aligned}
$$

Here, the integral over energy runs along the real axis, while the poles of Green's function $G_{ \pm}^{(0) R, A}$ are located in the complex $\varepsilon$ plane near the real axis. We can assume that temperature $T$ is very small but finite, so that all the poles of Fermi function $f(\varepsilon)$ are located at a finite distance from the real axis, $\varepsilon_{n}=\varepsilon_{F}+i(2 n+1) \pi T$.

Using Eq. (8) as definition of $G_{ \pm}^{(0) R}$ and substituting it to Eq. (A1) along with $G_{ \pm}^{(0) A}=\left(G_{ \pm}^{(0) R}\right)^{*^{*}}$, we present Eq. (A1) as 


$$
\begin{aligned}
\sigma_{y x}^{\mathrm{II}=} & -\frac{i e^{2} \alpha^{2} h}{2 \pi} \int \frac{d^{2} k}{(2 \pi)^{2}} \frac{1}{\lambda_{k}} \\
& \times \int_{-\infty}^{\infty} f(\varepsilon) d \varepsilon\left[\frac{1}{\left(\varepsilon-E_{k-}+i 0^{+}\right)\left(\varepsilon-E_{k+}+i 0^{+}\right)^{2}}\right. \\
& -\frac{1}{\left(\varepsilon-E_{k+}+i 0^{+}\right)\left(\varepsilon-E_{k-}+i 0^{+}\right)^{2}} \\
& -\frac{1}{\left(\varepsilon-E_{k-}-i 0^{+}\right)\left(\varepsilon-E_{k+}-i 0^{+}\right)^{2}} \\
& \left.+\frac{1}{\left(\varepsilon-E_{k+}-i 0^{+}\right)\left(\varepsilon-E_{k-}-i 0^{+}\right)^{2}}\right] .
\end{aligned}
$$

The integral over $\varepsilon$ contains simple and double poles, related to the Green's functions, and all of them can be shifted to the real axis. Correspondingly, the integration contour should be deformed to encircle each singularity in the complex plane. Thus, the contour would consist of some lines at the real axis and half circles around the poles. Each of the half circles gives half of the whole residue associated with the pole. Only the contribution of poles give real $\sigma_{y x}$. Thus, we take into account only this contribution. Then, we find

$$
\begin{aligned}
\sigma_{y x}^{\mathrm{II}}= & -\frac{i e^{2} \alpha^{2} h}{2 \pi} \int \frac{d^{2} k}{(2 \pi)^{2}} \frac{1}{\lambda_{k}} \int_{-\infty}^{\infty} d \varepsilon\left\{f ( \varepsilon ) \left[4 \frac{-i \pi \delta\left(\varepsilon-E_{k-}\right)}{\left(E_{k-}-E_{k+}\right)^{2}}\right.\right. \\
& \left.-4 \frac{-i \pi \delta\left(\varepsilon-E_{k+}\right)}{\left(E_{k+}-E_{k-}\right)^{2}}\right]+\frac{\partial f(\varepsilon)}{\partial \varepsilon}\left[2 \frac{-i \pi \delta\left(\varepsilon-E_{k+}\right)}{E_{k+}-E_{k-}}\right. \\
& \left.\left.+2 \frac{i \pi \delta\left(\varepsilon-E_{k-}\right)}{E_{k-}-E_{k+}}\right]\right\} .
\end{aligned}
$$

As we see from Eq. (A3), $\sigma_{y x}^{\mathrm{II}}$ contains the terms with $(\partial f / \partial \varepsilon)$ related to the contribution from the Fermi surface. Then, we obtain

$$
\sigma_{y x}^{\mathrm{II}-\text { clean }}=\sigma_{y x}^{\text {clean }}-\sigma_{y x}^{\mathrm{I} \text {-clean }},
$$

where

$$
\sigma_{y x}^{\text {clean }}=4 e^{2} \alpha^{2} h \int \frac{d^{2} k}{(2 \pi)^{2}} \frac{f\left(E_{k+}\right)-f\left(E_{k-}\right)}{\left(E_{k+}-E_{k-}\right)^{3}}
$$

and

$$
\begin{aligned}
\sigma_{y x}^{\text {I-clean }}= & -2 e^{2} \alpha^{2} h \int \frac{d^{2} k}{(2 \pi)^{2}}\left[\left(-\frac{\partial f\left(E_{k+}\right)}{\partial \varepsilon}\right)\right. \\
& \left.+\left(-\frac{\partial f\left(E_{k-}\right)}{\partial \varepsilon}\right)\right] \frac{1}{\left(E_{k+}-E_{k-}\right)^{2}} .
\end{aligned}
$$

Equation (A5) is the well-known formula for intrinsic Hall conductivity in the clean limit. It is related to all filled states (Fermi sea).

As follows from Eq. (A4), the contribution to $\sigma_{y x}^{\mathrm{II}-\text { clean }}$ from the Fermi surface defined as $\sigma_{y x}^{\mathrm{I} \text {-clean }}$ partly compensates the intrinsic Hall conductivity $\sigma_{y x}^{\text {clean }}$. Calculation of integral (A5) leads to

$$
\sigma_{y x}^{\text {clean }}=-\frac{e^{2}}{4 \pi}\left[1-\frac{h}{\lambda_{-}}-\Theta\left(\varepsilon_{F}-h\right)\left(1-\frac{h}{\lambda_{+}}\right)\right] .
$$

Using Eqs. (A6) and (10), we find

$$
\sigma_{y x}^{\mathrm{I} \text {-clean }}=-\frac{e^{2} \alpha^{2} h}{4 \pi}\left(\frac{\nu_{+}}{\lambda_{+}^{2}} \Theta\left(\varepsilon_{F}-h\right)+\frac{\nu_{-}}{\lambda_{-}^{2}}\right) .
$$

From Eq. (A4), Eqs. (A7) and (A8) follow Eq. (23).

Here, $\sigma_{y x}^{\mathrm{II}}$ corresponds to Streda's definition. ${ }^{20} \mathrm{~A}$ different method of calculating the conductivity has been used in Ref. 12. In principle, both methods lead to the same final result for the conductivity, as was recently demonstrated for the two-dimensional Dirac model. However, the contribution to the conductivity from the filled states, which was identified as $\sigma_{y x}^{\mathrm{II}}$ in Ref. 12, corresponds only to the "Fermi sea" term of Eq. (A5) which corresponds to the clean limit calculation of the anomalous Hall conductivity as in Ref. 11. In turn, the contribution $\sigma_{y x}^{\mathrm{I} \text {-clean }}$ related to the Fermi surface but not affected by impurities was included into the $\sigma_{y x}^{\mathrm{I}}$ of Ref. 12 (the vertex correction should not appear in this term as was later pointed out in Ref. 10). Hence, although initially the $\sigma_{y x}^{\mathrm{I}}$ and $\sigma_{y x}^{\mathrm{II}}$ in Ref. 12 are defined as we do here by Eqs. (4) and (5) in that article, their actual definitions were changed to terms only including Fermi sea integrals and Fermi surface integrals and hence not defined by Eqs. (4) and (5). Their result, when corrected by the subtle inclusion of the vertex correction of the clean term in $\sigma_{y x}^{\mathrm{I}}$ and additional vertex terms ignored in their Sec. III, agrees with ours here. We should emphasize here this difference of notations of $\sigma_{y x}^{\mathrm{I}}$ and $\sigma_{y x}^{\mathrm{II}}$ to avoid a possible misunderstanding.

\section{Calculation of $\sigma_{y x}^{\mathrm{II}}$ using the eigenstates}

Alternatively, one can also use the disorder free eigenstates of the Rashba Hamiltonian [Eq. (4)],

$$
| \pm\rangle=e^{ \pm i \phi / 2}\left(\begin{array}{c} 
\pm i e^{-i \phi / 2}\left(\cos \frac{\gamma}{2} \pm \sin \frac{\gamma}{2}\right) \\
e^{i \phi / 2}\left(\cos \frac{\gamma}{2} \mp \sin \frac{\gamma}{2}\right)
\end{array}\right) \text {, }
$$

where $\sin \gamma=h / \lambda$ and $\tan \phi=k_{y} / k_{x}$ to calculate the clean limit of $\sigma_{y x}^{\text {II-clean }}$ as the difference $\sigma_{y x}^{\text {II-clean }}=\sigma_{y x}^{\text {clean }}-\sigma_{y x}^{\text {I-clean }}$ given in Eq. (A4).

In the clean limit, one can calculate $\sigma_{y x}$ as

$$
\begin{aligned}
\sigma_{y x}^{\text {clean }} & =\frac{e^{2}}{m^{2} V} \sum_{\mathbf{k}, n \neq n^{\prime}} \frac{\left(f_{n^{\prime}, k}-f_{n, k}\right) \operatorname{Im}\left[\left\langle n^{\prime} k\left|\hat{p}_{x}\right| n k\right\rangle\left\langle n k\left|\hat{p}_{y}\right| n^{\prime} k\right\rangle\right]}{\left(E_{n k}-E_{n^{\prime} k}\right)^{2}} \\
& =-\frac{e^{2}}{(2 \pi)^{2}} \int d \mathbf{k} \sum_{n} f_{n, k} 2 \operatorname{Im}\left\langle\frac{\partial u_{n, k}}{\partial k_{y}} \mid \frac{\partial u_{n, k}}{\partial k_{x}}\right\rangle \\
& =-\frac{e^{2}}{(2 \pi)^{2}} \sum_{n} \int_{C} d \mathbf{k} \cdot\left\langle n k\left|i \frac{\partial}{\partial \mathbf{k}}\right| n k\right\rangle \\
& =-\frac{e^{2}}{(2 \pi)^{2}} \sum_{n} \int_{0}^{2 \pi} d \phi\left\langle n k\left|i \frac{\partial}{\partial \phi}\right| n k\right\rangle,
\end{aligned}
$$

where, for the eigenstates given in Eq. (A9), one finds 


$$
\left\langle \pm, k\left|i \frac{\partial}{\partial \phi}\right| \pm, k\right\rangle=\mp \frac{\lambda-h}{2 \lambda}
$$

yielding exactly expression (A7), for $\sigma_{y x}^{\text {clean }}$.

The clean limit contribution of $\sigma_{y x}^{\mathrm{I}}$ can be calculated as follows:

$$
\begin{aligned}
\sigma_{y x}^{\text {I-clean }} & =\frac{e^{2}}{2 \pi V} \operatorname{Tr}\left[\hat{v}_{y} G^{(0) R}\left(\epsilon_{F}\right) \hat{v}_{x} G^{(0) A}\left(\epsilon_{F}\right)\right] \\
& =\frac{e^{2}}{2 \pi V} \sum_{\mathbf{k}, s, s^{\prime}}\left\langle s\left|v_{y}\right| s^{\prime}\right\rangle G_{s^{\prime}}^{(0) R}\left\langle s^{\prime}\left|v_{x}\right| s\right\rangle G_{s}^{(0) A} .
\end{aligned}
$$

Substituting the $| \pm\rangle$ eigenstates [Eq. (A9)] of our Rashba Hamiltonian, one finds

$$
\begin{aligned}
\sigma_{y x}^{\text {I-clean }}= & \frac{e^{2}}{2 \pi V} \sum_{\mathbf{k}}\left(\left\langle+\left|v_{y}\right|-\right\rangle\left\langle-\left|v_{x}\right|+\right\rangle G_{-}^{(0) R} G_{+}^{(0) A}\right. \\
& \left.+\left\langle-\left|v_{y}\right|+\right\rangle\left\langle+\left|v_{x}\right|-\right\rangle G_{+}^{(0) R} G_{-}^{(0) A}\right) \\
= & \frac{e^{2}}{2 \pi V} \sum_{\mathbf{k}} 2 \operatorname{Im}\left[\left\langle+\left|v_{y}\right|-\right\rangle\left\langle-\left|v_{x}\right|+\right\rangle\right] \\
& \times\left(\frac{\pi \delta\left(\epsilon-E_{k-}\right)}{\epsilon-E_{k+}}-\frac{\pi \delta\left(\epsilon-E_{k+}\right)}{\epsilon-E_{k-}}\right) \\
= & \frac{e^{2}}{4 \pi}\left\langle\operatorname{Im}\left[\left\langle+\left|v_{y}\right|-\right\rangle\left\langle-\left|v_{x}\right|+\right\rangle\right]\right\rangle_{\phi}\left(\frac{\nu_{+}}{\lambda_{+}}+\frac{\nu_{-}}{\lambda_{-}}\right),
\end{aligned}
$$

where the subscript $\phi$ indicates averaging over the momentum angle $\phi$. For the Rashba model, one finds $\left\langle\operatorname{Im}\left[\left\langle+\left|v_{y}\right|-\right\rangle\right.\right.$ $\left.\left.\times\left\langle-\left|v_{x}\right|+\right\rangle\right]\right\rangle_{\phi}=-\alpha^{2} h / \lambda$, and therefore $\sigma^{\text {I-clean }}$ simplifies to expression (A8). This method therefore yields the same result for $\sigma_{y x}^{\mathrm{II}}$ as before, namely, the expression given in Eq. (23).

\section{Direct integration of $\sigma_{y x}^{\text {II }}$}

Instead of using the procedures described above, one can also directly integrate $\sigma^{\mathrm{II}}$ starting from the expression in Eq. (20) where one obtains the following after angular integration:

$$
\sigma_{y x}^{\mathrm{II}}=\frac{e^{2}}{4 \pi} \frac{1}{2 \pi} \int d k k \int_{-\infty}^{\infty} d \epsilon f(\epsilon) \frac{\alpha^{2} h}{\lambda_{k}} 4 \operatorname{Im}\left[G_{+}^{R} G_{-}^{R}\left(G_{+}^{R}-G_{-}^{R}\right)\right] .
$$

Now, performing the remaining integrals in the clean limit, i.e., using $\Gamma_{+}=\Gamma_{-}=\delta$, yields

$$
\begin{aligned}
\sigma_{y x}^{\mathrm{II}}= & -\frac{e^{2}}{4 \pi} \frac{1}{2 \pi} \int d k k \int_{-\infty}^{\infty} d \epsilon f(\epsilon) 4 \frac{\alpha^{2} h}{\lambda_{k}}\left(E_{k-}-E_{k+}\right) \\
& \times \operatorname{Im}\left[\frac{1}{\left(\epsilon-E_{k+}+i \delta\right)^{2}\left(\epsilon-E_{k-}+i \delta\right)^{2}}\right] \\
= & -\frac{e^{2}}{4 \pi} \frac{1}{2 \pi} \int d k k 4 \frac{\alpha^{2} h}{\lambda_{k}} \frac{1}{E_{k-}-E_{k+}} \\
& \times\left\{\pi \delta\left(E_{k+}-\epsilon_{F}\right)+\pi \delta\left(E_{k-}-\epsilon_{F}\right)\right.
\end{aligned}
$$

$$
\left.-\frac{2}{E_{k+}-E_{k-}} \operatorname{Im}\left[\ln \left(E_{k+}-\epsilon_{F}-i \delta\right)-\ln \left(E_{k-}-\epsilon_{F}-i \delta\right)\right]\right\} .
$$

Substituting $E_{k+}-E_{k-}=2 \lambda_{k}$ and using

$$
\begin{aligned}
\int d k \frac{k}{\lambda_{k}^{2}} \pi \delta\left(E_{k \pm}-\epsilon_{F}\right) & =\int_{ \pm h}^{\infty} d E_{k \pm} \frac{m}{\lambda_{k}\left|\lambda_{k} \pm \alpha^{2} m\right|} \pi \delta\left(E_{k \pm}-\epsilon_{F}\right) \\
& =\frac{\pi m}{\lambda_{ \pm}\left|\lambda_{ \pm} \pm \alpha^{2} m\right|} \Theta\left(\epsilon_{F}-E_{ \pm}^{\mathrm{min}}\right), \quad
\end{aligned}
$$

$$
\begin{aligned}
\int d k \frac{k}{\lambda_{k}^{3}} \ln \left(E_{k \pm}-\epsilon_{F}-i \delta\right)= & {\left[-\frac{\ln \left(E_{k \pm}-\epsilon_{F}-i \delta\right)}{\alpha^{2} \lambda_{k}}\right]_{0}^{\infty} } \\
& +\int_{0}^{\infty} \frac{d k}{\alpha^{2} \lambda_{k}} \frac{1}{E_{k \pm}-\epsilon_{F}-i \delta} \frac{d E_{k \pm}}{d k},
\end{aligned}
$$

and

$$
\begin{aligned}
& -\left[-\frac{\ln \left(E_{k+}-\epsilon_{F}-i \delta\right)}{\alpha^{2} \lambda_{k}}\right]_{0}^{\infty}+\left[-\frac{\ln \left(E_{k-}-\epsilon_{F}-i \delta\right)}{\alpha^{2} \lambda_{k}}\right]_{0}^{\infty} \\
& =-\frac{i \pi}{\alpha^{2} h} \Theta\left(h-\epsilon_{F}\right),
\end{aligned}
$$

$\sigma^{\mathrm{II}}$ simplifies to

$$
\begin{aligned}
\sigma_{y x}^{\mathrm{II}}= & \frac{e^{2}}{4 \pi} h\left(\frac{1}{m} \frac{1}{\lambda_{-}-\alpha^{2} m}-\frac{1}{m} \frac{1}{\lambda_{+}+\alpha^{2} m} \Theta\left(\epsilon_{F}-h\right)\right. \\
& \left.-\frac{1}{h} \Theta\left(h-\epsilon_{F}\right)\right) \\
= & -\frac{e^{2}}{4 \pi}\left(1-\frac{h}{\sqrt{h^{2}+2 \alpha^{2} m \epsilon_{F}+\left(\alpha^{2} m\right)^{2}}}\right) \Theta\left(h-\epsilon_{F}\right) .
\end{aligned}
$$

This, of course, is in full agreement with the prior results.

\section{APPENDIX B: INTEGRALS IN THE WEAK SCATTERING LIMIT}

In the weak scattering limit $\left(\Gamma, \Gamma_{z}\right.$ small), the integrals over two Green's functions simplify to

$$
\begin{aligned}
& \frac{1}{2 \pi} \int d k k f(k) G_{+}^{R}(k) G_{+}^{A}(k) \\
& \quad=\frac{1}{2 \pi} \int d k k f(k) \frac{1}{\epsilon_{F}-E_{k+}+i \Gamma_{+}} \frac{1}{\epsilon_{F}-E_{k+}-i \Gamma_{+}} \\
& \quad=\frac{1}{2 \pi} \int d E_{k+} \nu_{+} f\left(k\left(E_{k+}\right)\right) \frac{1}{\Gamma_{+}} \frac{\Gamma_{+}}{\left(E_{k+}^{2}-\epsilon_{F}^{2}\right)^{2}+\Gamma_{+}^{2}} \\
& \approx \frac{\nu_{+} f\left(k_{+}\right)}{2 \Gamma_{+}}
\end{aligned}
$$




$$
\frac{1}{2 \pi} \int d k k f(k) G_{-}^{R}(k) G_{-}^{A}(k) \approx \frac{\nu_{-} f\left(k_{-}\right)}{2 \Gamma_{-}},
$$

analogously, and

$$
\begin{aligned}
& \frac{1}{2 \pi} \int d k k f(k) G_{+}^{R}(k) G_{-}^{A}(k) \\
&=\frac{1}{2 \pi} \int d k k f(k) \frac{1}{\epsilon_{F}-E_{k+}+i \Gamma_{+}} \frac{1}{\epsilon_{F}-E_{k-}-i \Gamma_{-}} \\
& \approx \frac{1}{2 \pi} \int d k k f(k)\left(\frac{1}{\epsilon_{F}-E_{k+}}-i \pi \delta\left(\epsilon_{F}-E_{k+}\right)\right) \\
& \times\left(\frac{1}{\epsilon_{F}-E_{k-}}+i \pi \delta\left(\epsilon_{F}-E_{k-}\right)\right) \\
& \approx \frac{1}{2 \pi} \int d k k f(k) \frac{1}{\epsilon_{F}-\epsilon_{k}-\lambda_{k}} \frac{1}{\epsilon_{F}-\epsilon_{k}+\lambda_{k}} \\
&+ \frac{i}{2} \int d k k f(k)\left(\delta\left(\epsilon_{F}-\epsilon_{k}+\lambda_{k}\right) \frac{1}{\epsilon_{F}-\epsilon_{k}-\lambda_{k}}\right. \\
&-\left.\delta\left(\epsilon_{F}-\epsilon_{k}-\lambda_{k}\right) \frac{1}{\epsilon_{F}-\epsilon_{k}+\lambda_{k}}\right),
\end{aligned}
$$

yielding

$$
\begin{aligned}
\frac{1}{2 \pi} \int d k k f(k)\left[G_{+}^{R}(k) G_{-}^{A}(k)-G_{-}^{R}(k) G_{+}^{A}(k)\right] \\
\approx i \int d E_{k-} \frac{\nu_{-} f\left(k\left(E_{k-}\right)\right) \delta\left(\epsilon_{F}-E_{k-}\right)}{\epsilon_{F}-E_{k-}-2 \lambda_{k\left(E_{k-}\right)}} \\
-i \int d E_{k+} \frac{\nu_{+} f\left(k\left(E_{k+}\right)\right) \delta\left(\epsilon_{F}-E_{k+}\right)}{\epsilon_{F}-E_{k+}+2 \lambda_{k\left(E_{k+}\right)}} \\
=-\frac{i}{2}\left(\frac{\nu_{+} f\left(k_{+}\right)}{\lambda_{+}}+\frac{\nu_{-} f\left(k_{-}\right)}{\lambda_{-}}\right) .
\end{aligned}
$$

Now, we find the following for the integrals $I_{1}, I_{2}, I_{3}$, and $I_{4}$ in the weak scattering limit:

$$
\begin{aligned}
I_{1}= & \frac{1}{2 \pi} \int d k k\left(G_{0}^{R} G_{0}^{A}-G_{z}^{R} G_{z}^{A}\right) \\
= & \frac{1}{4} \frac{1}{2 \pi} \int d k k\left(\left(G_{+}^{R} G_{+}^{A}+G_{-}^{R} G_{-}^{A}+G_{+}^{R} G_{-}^{A}+G_{-}^{R} G_{+}^{A}\right)\right. \\
& \left.-\frac{\lambda_{k}^{2}\left(h^{2}+\Gamma_{z}^{2}\right)}{\lambda_{k}^{4}+h^{2} \Gamma_{z}^{2}}\left(G_{+}^{R} G_{+}^{A}+G_{-}^{R} G_{-}^{A}-G_{+}^{R} G_{-}^{A}-G_{-}^{R} G_{+}^{A}\right)\right)
\end{aligned}
$$

$$
\begin{aligned}
& \approx \frac{1}{4} \frac{1}{2 \pi} \int d k k\left(1-\frac{h^{2}}{\lambda_{k}^{2}}\right)\left(G_{+}^{R} G_{+}^{A}+G_{-}^{R} G_{-}^{A}\right) \\
& \approx \frac{1}{8}\left[\left(1-\frac{h^{2}}{\lambda_{+}^{2}}\right) \frac{\nu_{+}}{\Gamma_{+}}+\left(1-\frac{h^{2}}{\lambda_{-}^{2}}\right) \frac{\nu_{-}}{\Gamma_{-}}\right] \\
& I_{2}=\frac{1}{2 \pi} \int d k k\left(G_{0}^{R} G_{z}^{A}-G_{z}^{R} G_{0}^{A}\right) \\
& =-\frac{1}{2} \frac{1}{2 \pi} \int d k k \frac{\lambda_{k}}{\lambda_{k}^{4}+\Gamma_{z}^{2} h^{2}}\left[h\left(\lambda_{k}^{2}+\Gamma_{z}^{2}\right)\left(G_{-}^{R} G_{+}^{A}-G_{+}^{R} G_{-}^{A}\right)\right. \\
& \left.+i \Gamma_{z}\left(h^{2}-\lambda_{k}^{2}\right)\left(G_{+}^{R} G_{+}^{A}-G_{-}^{R} G_{-}^{A}\right)\right] \\
& \approx-\frac{1}{2} \frac{1}{2 \pi} \int d k k \frac{1}{\lambda_{k}^{3}}\left[h \lambda_{k}^{2}\left(-G_{+}^{R} G_{-}^{A}+G_{-}^{R} G_{+}^{A}\right)\right. \\
& \left.+i \Gamma_{z}\left(h^{2}-\lambda_{k}^{2}\right)\left(G_{+}^{R} G_{+}^{A}-G_{-}^{R} G_{-}^{A}\right)\right] \\
& \approx-\frac{i}{4}\left(\frac{\nu_{+} h}{\lambda_{+}^{2}}+\frac{\nu_{-} h}{\lambda_{-}^{2}}+\frac{\Gamma_{z}}{\Gamma_{+}} \frac{\nu_{+}\left(h^{2}-\lambda_{+}^{2}\right)}{\lambda_{+}^{3}}-\frac{\Gamma_{z}}{\Gamma_{-}} \frac{\nu_{-}\left(h^{2}-\lambda_{-}^{2}\right)}{\lambda_{-}^{3}}\right), \\
& I_{3}=\frac{1}{2 \pi} \int d k \frac{k^{2}}{2 m}\left(\widetilde{G}_{y}^{R} G_{z}^{A}-G_{z}^{R} \widetilde{G}_{y}^{A}\right) \\
& =-\frac{i}{2} \frac{1}{2 \pi} \int d k k \frac{k^{2}}{2 m} \frac{\alpha \Gamma_{z} \lambda_{k}^{2}}{\lambda_{k}^{4}+\Gamma_{z}^{2} h^{2}} \\
& \times\left(G_{+}^{R} G_{+}^{A}+G_{-}^{R} G_{-}^{A}-G_{+}^{R} G_{-}^{A}-G_{-}^{R} G_{+}^{A}\right) \\
& \approx-\frac{i}{2} \frac{1}{2 \pi} \int d k k \frac{k^{2}}{2 m} \frac{\alpha \Gamma_{z}}{\lambda_{k}^{2}}\left(G_{+}^{R} G_{+}^{A}+G_{-}^{R} G_{-}^{A}\right) \\
& \approx-\frac{i}{4} \alpha \Gamma_{z}\left[\epsilon_{F}\left(\frac{\nu_{+}}{\Gamma_{+} \lambda_{+}^{2}}+\frac{\nu_{-}}{\Gamma_{-} \lambda_{-}^{2}}\right)-\frac{\nu_{+}}{\Gamma_{+} \lambda_{+}}+\frac{\nu_{-}}{\Gamma_{-} \lambda_{-}}\right], \\
& I_{4}=\frac{1}{2 \pi} \int d k \frac{k^{2}}{2 m}\left(G_{0}^{R} \widetilde{G}_{y}^{A}+\widetilde{G}_{y}^{R} G_{0}^{A}\right) \\
& =-\frac{1}{2} \frac{1}{2 \pi} \int d k k \frac{k^{2}}{2 m} \frac{\alpha \lambda_{k}}{\lambda_{k}^{4}+\Gamma_{z}^{2} h^{2}}\left[\lambda_{k}^{2}\left(G_{+}^{R} G_{+}^{A}-G_{-}^{R} G_{-}^{A}\right)\right. \\
& \left.+i \Gamma_{z} h\left(G_{-}^{R} G_{+}^{A}-G_{+}^{R} G_{-}^{A}\right)\right] \\
& \approx-\frac{1}{2} \frac{1}{2 \pi} \int d k k \frac{k^{2}}{2 m} \frac{\alpha}{\lambda_{k}}\left(G_{+}^{R} G_{+}^{A}-G_{-}^{R} G_{-}^{A}\right) \\
& \approx-\frac{1}{4} \alpha\left[\epsilon_{F}\left(\frac{\nu_{+}}{\Gamma_{+} \lambda_{+}}-\frac{\nu_{-}}{\Gamma_{-} \lambda_{-}}\right)-\left(\frac{\nu_{+}}{\Gamma_{+}}+\frac{\nu_{-}}{\Gamma_{-}}\right)\right] \text {. }
\end{aligned}
$$

${ }^{1}$ E. H. Hall, Philos. Mag. 10, 301 (1880); Philos. Mag. 12, 157 (1881).

${ }^{2}$ J. Sinova, T. Jungwirth, and J. Cerne, Int. J. Mod. Phys. B 18, 1083 (2004).

${ }^{3}$ R. Karplus and J. M. Luttinger, Phys. Rev. 95, 1154 (1954).
${ }^{4}$ J. Smit, Physica (Amsterdam) 21, 877 (1955).

${ }^{5}$ Note that the origin of the asymmetry of this scattering arises from the spin-orbit coupling present in the Bloch states and not from the very weak spin-orbit coupling contribution of the disorder potential as noted originally by Smit. When projecting a 
multiband system to an effective conduction band system, one can obtain a term that looks as if it arises from such a spin-orbit coupling part of the disorder potential, but it truly originates from spin-orbit coupling induced by the valence band states and the normal disorder that is felt by them.

${ }^{6}$ L. Berger, Phys. Rev. B 2, 4559 (1970).

${ }^{7}$ P. Nozieres and C. Lewiner, J. Phys. (Paris) 34, 901 (1973).

${ }^{8}$ W. Kohn and J. M. Luttinger, Phys. Rev. 108, 590 (1957).

${ }^{9}$ J. M. Luttinger, Phys. Rev. 112, 739 (1958).

${ }^{10}$ N. A. Sinitsyn, A. H. MacDonald, T. Jungwirth, V. K. Dugaev, and J. Sinova, Phys. Rev. B 75, 045315 (2007).

${ }^{11}$ D. Culcer, A. H. MacDonald, and Q. Niu, Phys. Rev. B 68, 045327 (2003).

${ }^{12}$ V. K. Dugaev, P. Bruno, M. Taillefumier, B. Canals, and C. Lacroix, Phys. Rev. B 71, 224423 (2005).

${ }^{13}$ N. A. Sinitsyn, Q. Niu, J. Sinova, and K. Nomura, Phys. Rev. B
72, 045346 (2005).

${ }^{14}$ S. Y. Liu and X. L. Lei, Phys. Rev. B 72, 195329 (2005).

${ }^{15}$ S. Y. Liu, N. J. M. Horing, and X. L. Lei, Phys. Rev. B 74, 165316 (2006).

${ }^{16}$ J. I. Inoue, T. Kato, Y. Ishikawa, H. Itoh, G. E. W. Bauer, and L. W. Molenkamp, Phys. Rev. Lett. 97, 046604 (2006).

${ }^{17}$ S. Onoda, N. Sugimoto, and N. Nagaosa, Phys. Rev. Lett. 97, 126602 (2006).

${ }^{18}$ M. F. Borunda, T. S. Nunner, T. Luck, N. A. Sinitsyn, C. Timm, J. Wunderlichl, T. Jungwirth, A. H. MacDonald, and J. Sinova, Phys. Rev. Lett. 99, 066604 (2007).

${ }^{19}$ N. A. Sinitsyn, Q. Niu, and A. H. MacDonald Phys. Rev. B 73, 075318 (2006).

${ }^{20}$ P. Streda, J. Phys. C 15, L717 (1982).

${ }^{21}$ A. A. Kovalev, K. Vyborny, and J. Sinova (unpublished).

${ }^{22}$ A. Crepieux and P. Bruno, Phys. Rev. B 64, 014416 (2001). 